\title{
Daytime Visibility Range Monitoring through use of a Roadside Camera
}

\author{
Nicolas Hautière, Erwan Bigorgne and Didier Aubert
}

\begin{abstract}
Based on a road meteorology standard, we present a roadside camera-based system able to detect daytime fog and to estimate the visibility range. Two detection algorithms, both based on a daytime fog model, are presented along with a process to combine their outputs. Unlike previous methods, the system takes into account the 3-D scene structure and filters the moving objects from the region of interest through use of a background modelling approach and detects the cause of the visibility reduction. The study of the system accuracy with respect to the camera characteristics leads to a specification of the characteristics of the camera required for the system. Some results obtained using a reduced-scale prototyping of the system are presented. Finally, an outlook to future works is given.
\end{abstract}

\section{INTRODUCTION}

The major part of all the information used in driving is visual [19]. Reduced visibility thus leads to accidents. Reductions in visibility may have a variety of causes, namely the geometry of the road scene, the presence of obstacles or adverse weather/lighting conditions. The presence of daytime fog and low visibility areas can be detected using in-vehicle cameras [13], [11]. This information is used in order to switch on/off fog lights, to adapt the operation range of optical sensors and associated signal processings [12] or to report low visibility conditions to a traffic center using probe vehicles [4].

However, to increase the driver's safety margin [1], giving him an opportunity to adapt his driving behavior accordingly, the resolution of classical in-vehicle cameras is too small. If they are located at places with recurrent foggy weather [17], roadside sensors may provide more reliable information to be communicated to vehicles using infrastructure to vehicles communication or displayed using variable message signs. Unfortunately, classical visibility sensors are expensive and may not be appropriate, because the small size of the diffusing volume of a scatterometer makes the measurements highly sensitive to non-homogeneities in the fog [10]. We propose to replace these sensors with a simple infrastructurebased camera. Some solutions have already been proposed.

Bush computes the highest edge in the image having a contrast above 5\% using a wavelet transform [5]. However, the presence of vertical objects, like a truck, in their region of

This work is supported by SAFESPOT, a project initiated by the European Commission in the FP6 under the IST theme (IST-4-026963-IP).

N. Hautière is with the Laboratoire Central des Ponts et Chaussées, 58 boulevard Lefebvre 75015 Paris, France nicolas.hautiere@lcpc.fr

E. Bigorgne is with VIAMETRIS, Maison de la Technopole, 6 rue Léonard de Vinci, 53000 Laval, France erwan.bigorgnedviametris. fr

D. Aubert is with LIVIC, INRETS/LCPC, bldg 824, 14 route de la Minière, 78000 Versailles, France didier. aubert@inret. . fr interest alters the results of the method. Second, the fact that the precision of their method highly depends on the camera characteristics is omitted in the article. Kwon has performed successfull visibility tests utilizing fixed distance targets and defined a visibility index called Relate Visibility [16]. However, deploying such targets at each camera location would be expensive. Nevertheless, such an approach is useful to calibrate other methods which do not need references. Hagiwara proposed a weighted intensity power spectra algorithm which compared favorably to manually extracted image's visibility [8]. Hallowell proposed an algorithm which examines natural edges within the image (the horizon, tree lines, roadways, buildings) and performs a comparison of each image with a historical composite image [9]. However, [16], [8] do not take into account the 3-D structure of the scene to compute their visibility indicator. Consequently, the method is sensitive to the presence of vertical objects in the scene. Moreover, the different methods do not detect the cause of the visibility reduction.

Based on a road meteorology standard [2], we propose a reference-free roadside camera-based sensor which not only estimates the visibility range in daytime but also detects that the visibility reduction is caused by fog, unlike previous methods. Unlike [9], [8], we take the 3-D scene structure into account by detecting the driving space area. Unlike [5], we filter objects in the region of interest and study the precision of the system with respect to the camera characteristics.

First, we present the requirements of an adequate visibility sensor and specify two detection algorithms to fulfill the functional requirements based on a daytime fog modelling. To correctly implement these algorithms, a background modelling approach is proposed, as well as a data fusion process to determine the visibility range. Then, we specify the camera in order to fulfill the requirements on the system accuracy. Finally, we present a reduced scale prototyping of the method as well as some results.

\section{VISIBILITY SENSOR REQUIREMENTS}

According to [2], the road visibility is defined as the horizontal visibility determined $1.2 \mathrm{~m}$ above the roadway. It may be reduced to less than $400 \mathrm{~m}$ by fog, precipitations or

TABLE I

VISIBILITY RANGES

\begin{tabular}{|c||c|}
\hline Visibility range index & Horizontal visibility distance $(\mathrm{m})$ \\
\hline 1 & 200 to 400 \\
2 & 100 to 200 \\
3 & 50 to 100 \\
4 & $<50$ \\
\hline
\end{tabular}


projections. Four visibility ranges are defined and are detailed in Table I. Based on these definitions, a visibility sensor should assign the visibility range to one of the four categories and detect the origin of the visibility reduction, i.e. it should detect fog, rain and projections. In this paper, we focus on daytime fog detection and visibility range estimation.

\section{DAYTIME FOG EFFECTS ON VISION}

\section{A. Visual Properties of Daytime Fog}

The attenuation of luminance through the atmosphere was studied by Koschmieder [18] who derived an equation relating the apparent luminance or radiance $L$ of an object located at distance $d$ to the intrinsic luminance $L_{0}$ :

$$
L=L_{0} e^{-k d}+L_{f}\left(1-e^{-k d}\right)
$$

where $k$ is the extinction coefficient of the atmosphere and $L_{f}$ is the atmospheric luminance. In the presence of fog, it corresponds to the background luminance on which the target can be detected. On the basis of this equation, Duntley [18] developed a contrast attenuation law, stating that a nearby object exhibiting contrast $C_{0}$ with the background will be perceived at distance $d$ with the following contrast:

$$
C=\left[\left(L-L_{f}\right) / L_{f}\right] e^{-k d}=C_{0} e^{-k d}
$$

This expression stands as a basis for the definition of a standard dimension called "meteorological visibility distance" $V_{m e t}$, i.e. the greatest distance at which a black object $\left(C_{0}=-\right.$ 1) of a suitable dimension can be seen in the sky on the horizon, with the threshold contrast set at 5\%. The meteorological visibility distance is thus a standard dimension which characterizes the opacity of a fog layer. This definition yields the following expression:

$$
V_{\text {met }}=-\log (0.05) / k \approx 3 / k
$$

\section{B. Camera Response}

Let us denote $f_{c}$ the camera response function [7]. With the notations of Fig. 1, the intensity $I$ of a pixel is the result of $f_{c}$ applied to the sum of the airlight $A$ and the direct transmission $T$, i.e $I=f_{c}(L)=f_{c}(T+A)$. If we assume

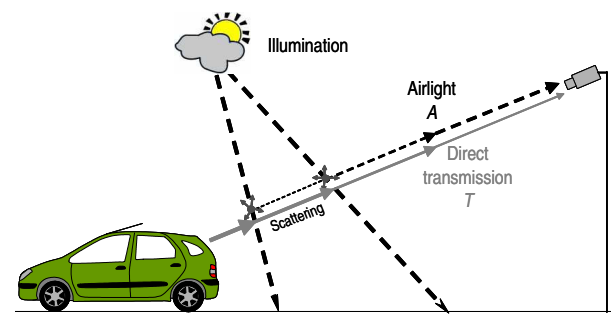

Fig. 1. Fog luminance is due to the scattering of daylight. Light coming from the sun and scattered by atmospheric particles towards the camera is the airlight $A$. It increases with the distance. The light emanating from the object $R$ is attenuated by scattering along the line of sight. Direct transmission $T$ of $R$ decreases with distance. that the conversion process between incident energy on the sensor array and the intensity in the image is linear:

$$
\begin{aligned}
I & =f_{c}(T)+f(A)=f_{c}\left(L_{0} e^{-k d}\right)+f_{c}\left(L_{f}\left(1-e^{-k d}\right)\right) \\
& =f_{c}\left(L_{0}\right) e^{-k d}+f_{c}\left(L_{f}\right)\left(1-e^{-k d}\right) \\
& =R e^{-k d}+A_{\infty}\left(1-e^{-k d}\right)
\end{aligned}
$$

where $R$ is the intrinsic intensity of the pixel, i.e. the intensity corresponding to the intrinsic luminance value of the corresponding scene point and $A_{\infty}$ is the background sky intensity.

\section{DETECTION ALGORITHMS}

\section{A. Sensor Modelling}

Fig. 2 shows the modelling of a camera within the road environment. In the image reference plane, the position of a pixel is given by its $(u, v)$ coordinates. The coordinates of the optical center in the image are denoted by $\left(u_{0}, v_{0}\right) . \theta$ denotes the pitch angle of the camera, while $v_{h}$ represents the vertical position of the horizon line. The intrinsic parameters of the camera are its focal length $f$, and the size $t_{p}$ of a pixel. We have also made use herein of $\alpha=\frac{f}{t_{p}}$. Based on [13], assuming that the road is locally planar, the distance $d$ can be expressed by:

$$
d=\lambda /\left(v-v_{h}\right)
$$

where $\lambda=\frac{H \alpha}{\cos (\theta)}$ and $v_{h}=v_{0}-\alpha \tan (\theta)$.

\section{B. Daytime Fog Detection}

1) Principle: Following a change of $d$ according to $v$ based on (5), (4) then becomes:

$$
I(v)=R-\left(R-A_{\infty}\right)\left(1-e^{-\frac{k \lambda}{v-v_{h}}}\right)
$$

By taking the second derivative of $I$ with respect to $v$, one obtains the following:

$$
\frac{d^{2} I}{d v^{2}}(v)=k \varphi(v) e^{-\frac{k \lambda}{v-v_{h}}}\left(\frac{k \lambda}{v-v_{h}}-2\right)
$$

where $\varphi(v)=\frac{\lambda\left(R-A_{\infty}\right)}{\left(v-v_{h}\right)^{3}}$. The equation $\frac{d^{2} I}{d v^{2}}=0$ has two solutions. The solution $k=0$ is of no interest. The only useful solution is given in (8):

$$
k=2\left(v_{i}-v_{h}\right) / \lambda
$$

where $v_{i}$ denotes the position of the inflection point of $I(v)$. In this manner, if $v_{i}>v_{h}$, daytime fog is detected and the parameter $k$ is obtained. We deduce $V_{\text {met }}$ using (3).

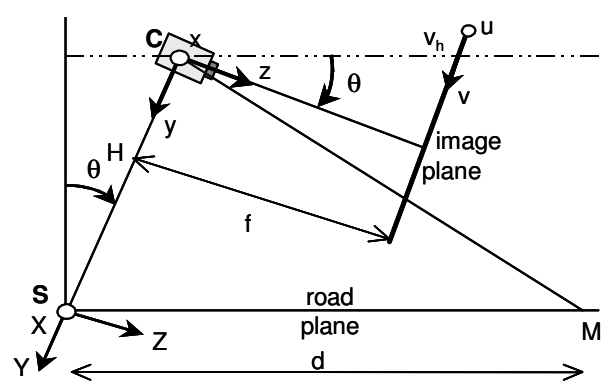

Fig. 2. Modelling of the camera within the road environment. $v_{h}$ : image line corresponding to the horizon line in the image. 
2) Implementation: A region within the image that displays minimal line-to-line gradient variation when browsed from bottom to top is identified thanks to a region growing process. A vertical band is then selected in the detected area. Finally, taking the median intensity of each segment yields the vertical variation of the intensity of the image and the position of the inflection point. Details of the method are given in [13]. It has been applied to a sample image in Fig. 3(a). Even if there are many vehicles in the original image, the method is able to circumvent them and to detect fog presence, as well as its density.

\section{Estimation of the Visibility Distance}

The previous method detects that the visibility is reduced by daytime fog and estimates its density. In the same way, methods dedicated to other meteorological phenomena quantification could be added. Nevertheless, to supervise these different methods, we need a generic method to estimate the visibility.

The principle of this approach is to compute the distance to the furthest visible point on the road surface. It fits well with the definition of $V_{\text {met }}$. We call it the mobilized visibility distance $V_{m o b}$. Based on [11], by using a $5 \%$ contrast threshold, $V_{m o b}$ is close to $V_{m e t}$.

A local contrast computation algorithm, based on Köhler's binarization technique and detailed in [11], is applied to the image to compute local contrasts above or equal to $5 \%$. The obtained contrast map contains objects of the road scene. A flat road may be assumed. As a matter of fact, along a topbottom scanning line of the local contrast map starting from the horizon line, objects encountered get closer to the camera. Consequently, the algorithm consists in finding the highest point in the contrast map having a local contrast above $5 \%$. We denote $v_{c}$ the corresponding image-line. The distance to this point can then be recovered using (5).

However, the image may also contain vertical objects, which do not respect the flat world assumption and alter the method. This is the case in Fig. 3(b), where the vehicle lights are detected higher in the image than the road surface elements. Another step is thus needed to filter the vertical objects and correctly estimate the visibility distance. We propose to achieve this task using a background modelling method in the next section.

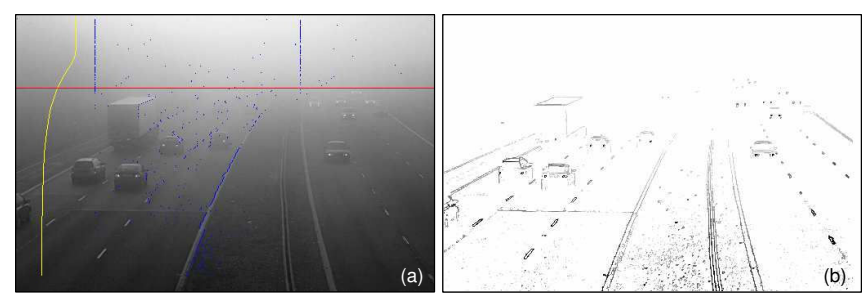

Fig. 3. (a) The vertical yellow curve represents the instantiation of (4); the horizontal red line represents the estimation of the visibility distance. The blue vertical segments represent the limits of the vertical band analyzed. (b) Map of local contrasts above $5 \%$.

\section{BACKGROUND MODELLING}

Both preceding methods need the road surface to run properly. Thanks to background modelling methods, it is possible to detect moving objects, i.e. the foreground, in video sequences by subtracting a background image from each new frame. In our application, we use the background image to compute the visibility distance and the foreground image to segment the driving space area.

\section{A. State of the art}

The simplest form of background modelling is a timeaveraged background image. This method suffers from many problems and requires a training period absent of foreground objects. In addition, the approach cannot cope with gradual illumination changes in the scene. Due to illumination changes and "long term" changes within the scene, it is necessary to constantly reestimate the background model. Many adaptive background-modelling methods have been proposed to deal with these slowly-changing signals. A comparison of different methods is proposed in [6]. One of the best methods has been proposed by Grimson [20] and uses an adaptive Gaussian mixture (MoG) model per pixel. The method we use is based on [20]. The differences lie in the update equations and the initialisation method, which are both described in [14].

\section{B. Adaptive Gaussian Mixture Model}

1) Principle: The method models each background pixel by a mixture of $K$ Gaussian distributions ( $K$ is classically a small number from 3 to 5). Different Gaussians are assumed to represent different intensities. The weight parameters of the mixture represent the time proportions that those intensities are in the scene. The background components are determined by assuming that the background contains $B$ highest probable intensities. The more probable background intensities are the ones which stay longer and are more static. Static single-intensity objects tend to form tight clusters in the intensity space while moving ones form wider clusters due to different reflecting surfaces during the movement. The measurement of this is called the fitness value. To allow the model to adapt to changes in illumination and to run in realtime, an update scheme is applied. It is based upon selective updating. Every new pixel value is checked against existing model components by decreasing fitness. The first matched model component is updated. If it finds no match, a new Gaussian component is added with the mean at that point, a large variance and a small weight.

2) Equations: Each pixel in the scene is modelled by a mixture of $K$ Gaussian distributions. The probability that a certain pixel has a value $X_{t}$ at time $t$ can be written as:

$$
P\left(X_{t}\right)=\sum_{j=1}^{K} \omega_{j} \eta\left(X_{t} ; \mu_{j}^{t}, \Sigma_{j}^{t}\right)
$$

where $\omega_{k}$ is the weight parameter of the $k^{\text {th }}$ Gaussian component. $\eta\left(X ; \mu_{k}, \Sigma_{k}\right)$ is the Normal distribution of $k^{t h}$ 
component represented by:

$$
\eta\left(X ; \mu_{k}, \Sigma_{k}\right)=\frac{1}{\sqrt{2 \pi\left|\Sigma_{k}\right|}} e^{-\frac{1}{2} \Sigma_{k}^{-1}\left(X-\mu_{k}\right)^{2}}
$$

where $\mu_{k}$ is the mean and $\Sigma_{k}=\sigma_{k}^{2}$ is the variance of the $k^{\text {th }}$ component. The $K$ distributions are ordered based on the fitness value $\frac{\omega_{k}}{\sigma_{k}}$ and the first $B$ distributions are used as a model of the scene background where $B$ is estimated as:

$$
B=\underset{b}{\operatorname{argmin}}\left(\sum_{j=1}^{b} \omega_{j}>T\right)
$$

The threshold $T$ is the minimal fraction of the background model. In other words, it is the minimum prior probability of observing a background pixel. Background subtraction is performed by marking as foreground any pixel that is more than 2.5 standard deviations away from any of the $B$ distributions. The first Gaussian component that matches the tested value is updated thanks to the following update equations:

$$
\begin{aligned}
\omega_{k}^{t} & =\left(1-\gamma_{1}\right) \omega_{k}^{t-1}+\gamma_{1}\left(M_{k}^{t}\right) \\
\mu_{k}^{t} & =(1-\rho) \mu_{k}^{t-1}+\rho X_{t} \\
\Sigma_{k}^{t} & =(1-\rho) \Sigma_{k}^{t-1}+\rho\left(X_{t}-\mu_{k}^{t}\right)^{2} \\
\rho & =\gamma_{1} \eta\left(X_{t} ; \mu_{k}^{t}, \Sigma_{k}^{t}\right) \\
\left(M_{k}^{t}\right) & =\left\{\begin{array}{l}
1 ; \text { if } \omega_{k} \text { is the first matched component } \\
0 ; \text { otherwise }
\end{array}\right.
\end{aligned}
$$

where $\omega_{k}$ is the weight for the $k^{t h}$ Gaussian component. $1 / \gamma_{1}$ defines the time constant which determines change. Only two parameters, $\gamma_{1}$ and $T$ need to be set.

\section{Driving Space Area Determination}

Thanks to the previous algorithm, we are able to split the foreground and the background in the current image. To implement detection methods, we now need to segment the driving space area in the background image. The driving space is the area where moving objects (car, pedestrians...) are able to travel. Consequently, this is the area where the flat world may be assumed. A naïve solution is to use an a priori mask of the road surface. However, due to perspective, some vertical objects (trees, urban lights, road signs) lie on this area, like in Fig. 4(a). To solve this problem, we propose to build a map $D$ which is the temporal accumulation of the foreground image, using:

$$
D_{t}=\left(1-\gamma_{2}\right) D_{t-1}+\gamma_{2} F_{t}
$$

where $\frac{1}{\gamma_{2}} \gg \frac{1}{\gamma_{1}}$ denotes again a time constant and $F$ the foreground image. A simple binarization is then applied to the map. In this way, we obtain the driving space area. Fig. 4(c) is the driving space before its binarization.

In some cases, we are only interested in a small part of the driving space area. For example, in Fig. 4(a), the tramway line is of no interest to us. A manual segmentation, e.g. Fig. 4(b), of the region of interest may be performed to obtain the final driving space area represented in Fig. 4(d).
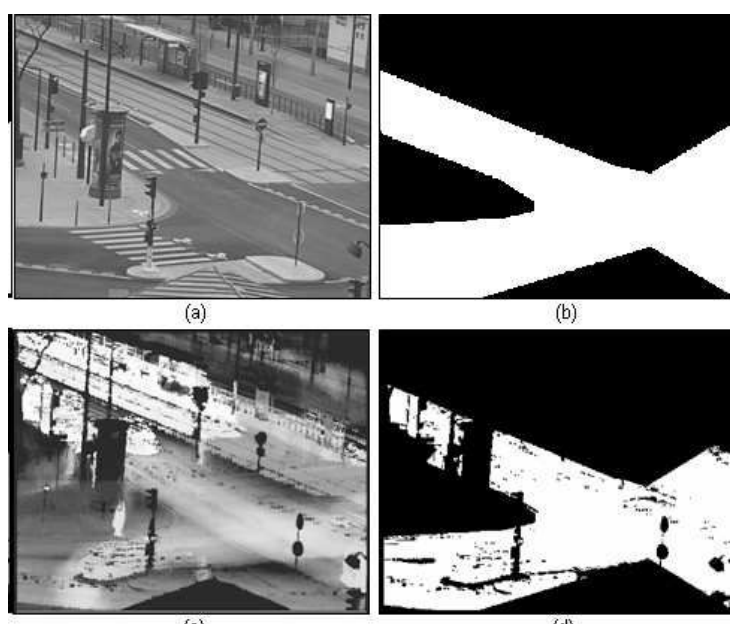

Fig. 4. (a) Background image of an urban intersection. (b) Manual segmentation of the driving space area. (c) Temporal accumulation of the moving objects in the foreground image. (d) Final driving space area which is the combination of images (b) and (c). Vertical objects like signs, trees, advertising are circumvented.

\section{Settings of the MoG for Visibility Range Monitoring}

Setting the parameters of the mixture of Gaussian is a crucial part of the algorithm. The temporal window must be big enough in order to compute a background image which does not contain moving objects on the driving space area. The temporal window must also be small enough to take into account the changes of visibility conditions.

Based on these considerations, we have chosen to set the temporal window approximatively equal to the average maximum time a moving object needs to cross the image, i.e. its smallest possible value. Such choice enables to take into account gradual changes of the visibility conditions. In Fig. 5, we have computed the background image using a short video sequence (14 s) of an intersection grabbed under foggy weather. The background model, shown in Fig. 5(b), does not contain moving objects, while fog density remains the same as in the original images, e.g. Fig. 5(a).

However, due to the luminance veil phenomenon, fog amplifies illumination changes which can be problematic in the background model computation. Indeed, we have seen in section V-A that major drawbacks of background computation approaches are the sensitivity to illumination changes. Some authors have developed approaches based on level sets computation [3] which are less sensitive to

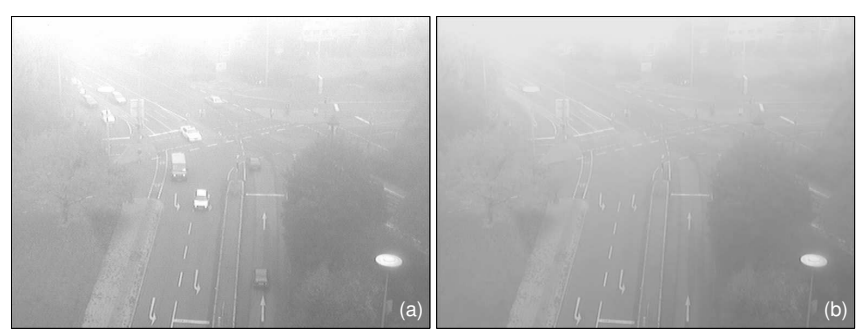

Fig. 5. (a) Sample of a 14 seconds video sequence grabbed under foggy weather [15]. (b) Resulting background model of the scene which includes the luminous veil caused by fog. 
illumination changes. In our case, it is not possible to use such a method because we need the entire road surface (textureless) to feed the detection algorithms adequately. We have thus developed another mitigation solution. Illumination changes create some artefacts in the background image, so that the number of its edges suddenly increases. We thus compute the number of edges, then its variations and we set a threshold above which the mixture of gaussian must be initialized again.

\section{VISIBILITY RANGE DETERMINATION}

Based on the generic method which estimates $V_{m o b}$ and Table I, the visibility range index $r$ can be determined. Thus, if $r>0$, a roadside alert may be launched. However, in the case of daytime fog, $V_{\text {met }}$ can also be estimated. Since $V_{\text {met }}$ and $V_{m o b}$ are close to each other, based on IV-C, a solution is to combine both indicators to reduce the measurements variance and obtain a single visibility descriptor at instant $k$ :

$$
V_{k}=\frac{\frac{V_{\text {met }_{k}}}{\Sigma_{V_{\text {met }_{k}}}}+\frac{V_{\text {mob }_{k}}}{\Sigma_{V_{\text {mob }_{k}}}}}{\frac{1}{\Sigma_{V_{\text {met }_{k}}}}+\frac{1}{\Sigma_{V_{\text {mob }_{k}}}}}=\frac{6 \lambda\left(v_{i}-v_{h}\right)+9 \lambda\left(v_{c}-v_{h}\right)}{4\left(v_{i}-v_{h}\right)^{2}+9\left(v_{c}-v_{h}\right)^{2}}
$$

$\Sigma_{V_{m e t}}$ and $\Sigma_{V_{m o b}}$ denote the variance of respectively $V_{m e t}$ and $V_{m o b}$ and are approximated as following, assuming that the variables are not correlated:

$$
\begin{aligned}
\Sigma_{V_{m e t}} & \approx \mathcal{V}_{I} \sum\left[\frac{\partial V_{m e t}}{\partial \lambda, v_{i}, v_{h}}\right]^{2}=\frac{9 \mathcal{V}_{I}}{4\left(v_{i}-v_{h}\right)^{2}} \\
\Sigma_{V_{m o b}} & \approx \mathcal{V}_{I} \sum\left[\frac{\partial V_{m o b}}{\partial \lambda, v_{c}, v_{h}}\right]^{2}=\frac{\mathcal{V}_{I}}{\left(v_{c}-v_{h}\right)^{2}}
\end{aligned}
$$

where $\mathcal{V}_{I}$ is the variance on the pixel value due to the digitalization of the pictures, assuming a gaussian centered distribution with a standard deviation of $1 / 2$. The variance $\Sigma_{k}$ of $V_{k}$ is then:

$$
\Sigma_{k}=\frac{9 \mathcal{V}_{I}}{4\left(v_{i}-v_{h}\right)^{2}+9\left(v_{c}-v_{h}\right)^{2}}
$$

One could also determine $r$ using $V_{k}=\min \left(V_{m o b}, V_{m e t}\right)$. The algorithm to determine $r$ is thus an open issue.

Nevertheless, to reduce the risk of false alarms, a temporal averaging of the measurements has to be carried out. We propose to use a simple linear Kalman filter [21] which allows to compute an weighted iterative least-squares regression. Since we cannot predict the variations of the visibility range, we adopt the simplest evolution model and introduce a process noise $Q$ to progressively forget the past measurements:

$$
\begin{aligned}
\hat{V}_{k}^{-} & =\hat{V}_{k-1} \\
P_{k}^{-} & =P_{k-1}+Q
\end{aligned}
$$

Using (17), the correction step of the filter is as following:

$$
\begin{aligned}
K_{k} & =P_{k}^{-} /\left(P_{k}^{-}+\Sigma_{k}\right) \\
\hat{V}_{k} & =\hat{V}_{k-1}+K_{k}\left(V_{k}-\hat{V}_{k-1}\right) \\
P_{k} & =\left(1-K_{k}\right) P_{k}^{-}
\end{aligned}
$$

where $\Sigma_{k}$ denotes the variance of $V_{k}$. The single parameter to tune is $Q$.

\section{CAMERA SPECIFICATIONS}

First, according to the sensor requirements given in section II, the visibility system shall detect visibility up to $d_{\max }$ (400 $\mathrm{m}$ in our case). Thanks to (5), we can compute the surface covered by a pixel for different typical sensor configurations at the distance $d$ :

$$
\Delta(d)=\frac{\lambda}{\left\lfloor v_{h}+\frac{\lambda}{d}\right\rfloor-v_{h}}-\frac{\lambda}{\left\lceil v_{h}+\frac{\lambda}{d}\right\rceil-v_{h}}
$$

where $\lfloor x\rfloor$ designates the whole part of $x$ and $\lceil x\rceil$ the integer greater than or equal to $x$. We propose this surface to be lower than $10 \%$ of $d_{\max }$, (40 $\mathrm{m}$ in our case):

$$
\Delta\left(d_{\max }\right)<0.1 d_{\max }
$$

Second, the system must detect fog. Based on section IV$\mathrm{B}$, the horizon line must be in the image. Third, the visibility system shall detect low visibilities lower than $d_{\text {min }}(50 \mathrm{~m}$ in our case). To run correctly, the corresponding location of the inflection point must lie in the upper part of the image, i.e. $v_{i}$ must be lower than $v_{0}$. Consequently, additional constraints on the sensor are as following:

$$
\begin{aligned}
v_{h} & >0 \\
v_{h}+3 \lambda / d_{\min } & >v_{0}
\end{aligned}
$$

From constraints (25) and (26), we obtain the following inequation with respect to $\theta$ :

$$
\sin ^{-1}\left(H / 3 d_{\text {min }}\right)<\theta<\tan ^{-1}\left(v_{0} / \alpha\right)
$$

The admissible solutions of (27) can then be used to solve (24). Some technical solutions are given in Table II. To choose between the different solutions, we have computed the parameter denoted $\chi$ which gives the magnification of the camera with respect to its pitch angle. We have thus purchased cameras corresponding to the third solution of the Table II.

TABLE II

TECHNiCAL SOLUTIONS

\begin{tabular}{|c||c|c|c|}
\hline$D$ (inch) & $1 / 3$ & $2 / 3$ & $1 / 2$ \\
\hline$H(\mathrm{~m})$ & $5-6$ & $5-6$ & 6 \\
\hline$f(\mathrm{~mm})$ & 4.2 & 4.8 & 4.5 \\
\hline$t_{p}(\mu \mathrm{m})$ & 4.65 & 6.45 & 4.65 \\
\hline $\operatorname{dim}_{y}($ pix $)$ & 1040 & 1024 & 1360 \\
\hline$\theta$ (degree) & $31-38$ & $29-64$ & $28-29$ \\
\hline \hline$\chi=f / t_{p} \cos (\theta)$ & 1023 & 851 & 1096 \\
\hline
\end{tabular}

\section{REDUCED SCALE PROTOTYPING}

Currently, we do not have at our disposal video sequences of fog which fulfill our system requirements. Consequently, the system, summarized in Fig. 6, has only been tested on a reduced scale model using a glass tank in which some scattering medium is injected using a fog machine. The sun is replaced by two strong light projectors. The sky is replaced by some scattering material put on the roof of the aquarium. Some remote control cars are used to create road traffic.

The results obtained using a video sequence with a moving car inside the tank are given in Fig. 7 and illustrated in Fig. 8. After the initialization period, the system provides stable results despite the presence of the car motion. 


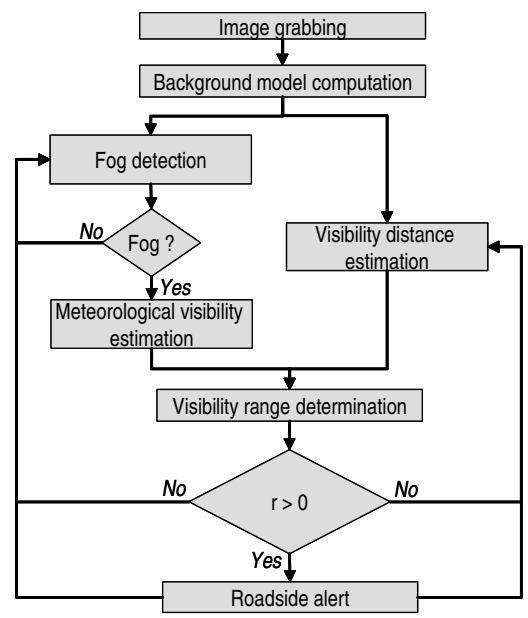

Fig. 6. Overview of the proposed daytime visibility range algorithm.
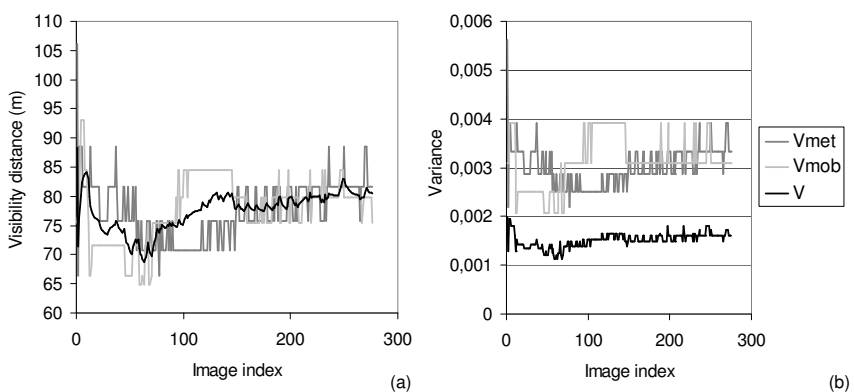

Fig. 7. (a) Estimation of the different visibility distances. $V$ is the final result using Kalman filtering $\left(Q=10^{-4}\right)$; (b) Variances of the different measurements. Settings of the MoG: $T=7 ; 1 / \gamma_{1}=200$.

\section{FUTURE WORKS}

First, we would like to test the system using a full scale installation, which should be soon the case in the European SAFESPOT project. Since the specified camera is a highresolution one, we would also like to test the system using a classical CCTV camera and see if the degradation of the performances is significant or not. Finally, we are extending the system to handle night fog situations.

\section{CONCLUSION}

In this paper, we presented first the requirements of an adequate visibility sensor and specified two detection algorithms to fulfill the functional requirements based on a daytime fog modelling. To enable these detection methods

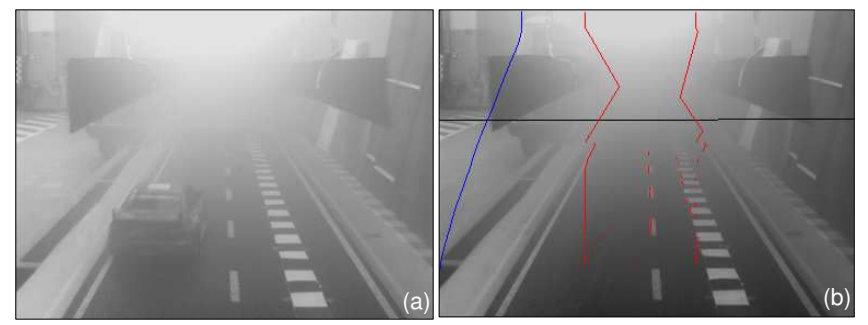

Fig. 8. (a) Sample image of the reduced scale model with a remote control car which creates some motion in the video sequence. (b) Sample result of the fog detection algorithm using the computed background image. The car no longer appears in the image. to run properly and to segment the driving space area, a background modelling approach was proposed using a MoG approach, as well as a data fusion process to determine the visibility range. Then, we specified a camera in order to fulfill the requirements on the system accuracy. Finally, we presented a reduced scale prototype of the method as well as some results. Perspectives and future research directions are indicated.

\section{REFERENCES}

[1] SAFESPOT - cooperative systems for road sadefty. http://www.safespot-eu.org/, 2006-2010.

[2] AFNOR. Road meteorology - gathering of meteorological and road data - terminology. NF P 99-320, April 1998.

[3] D. Aubert, F. Guichard, and S. Bouchafa. Time-scale change detection applied to real time abnormal stationarity monitoring. Real-Time Imaging, 10(1):9-22, 2004.

[4] C. Boussard, N. Hautière, and B. d'André Novel. Vision guided by vehicle dynamics for onboard estimation of the visibility range. In IFAC Symposium on Intelligent Autonomous Vehicles, Toulouse, France, September 3-5 2007.

[5] C. Bush and E. Debes. Wavelet transform for analyzing fog visibility. IEEE Intelligent Systems, 13(6):66-71, November/December 1998.

[6] S.-C. Cheung and C. Kamath. Robust techniques for background subtraction in urban traffic video. In Video Communications and Image Processing, SPIE Electronic Imaging, pages 881-892, January 2004.

[7] M. Grossberg and S. Nayar. Determining the camera response from images: What is knowable? IEEE Transactions on Pattern Recognition and Machine Intelligence, 25(11):1455-1467, November 2003.

[8] T. Hagiwara, Y. Ota, Y. Kaneda, Y. Nagata, and K. Araki. A method of processing CCTV digital images for poor visibility identification. In Proc. 85th Transportation Research Board Annual Meeting, 2006.

[9] R. Hallowell, M. Matthews, and P. Pisano. An automated visibility detection algorithm utilizing camera imagery. In 23rd Conference on IIPS, 87th AMS Annual Meeting, San Antonio, Texas, USA, January 2007.

[10] N. Hautière, R. Labayrade, and D. Aubert. Estimation of the visibility distance by stereovision: a generic approach. IEICE Transactions on Information and Systems, E89-D(7):2084-2091, July 2006.

[11] N. Hautière, R. Labayrade, and D. Aubert. Real-time disparity contrast combination for onboard estimation of the visibility distance. IEEE Transactions on Intelligent Transportation Systems, 7(2):201212, June 2006.

[12] N. Hautière, J.-P. Tarel, and D. Aubert. Towards fog-free in-vehicle vision systems through contrast restoration. In IEEE Computer Society Conference on Computer Vision and Pattern Recognition, Minneapolis, USA, June 2007.

[13] N. Hautière, J.-P. Tarel, J. Lavenant, and D. Aubert. Automatic fog detection and estimation of visibility distance through use of an onboard camera. Machine Vision and Applications Journal, 17(1):820, April 2006.

[14] P. KadewTraKuPong and R. Bowden. An improved adaptive background mixture model for real-time tracking with shadow detection. In 2nd European Workshop on Advanced Video-Based Surveillance Systems, Kingston, UK, 2001.

[15] KOGS/IAKS Universität Karlsruhe. Traffic image sequences and 'marbled block' sequence. http://i21www.ira.uka.de/.

[16] T. M. Kwon. Atmospheric visibility measurements using video cameras: Relative visibility. Technical report, University of Minnesota Duluth, July 2004.

[17] K. MacHutchon and A. Ryan. Fog detection and warning, a novel approach to sensor location. In IEEE African Conference, Cap Town, South Africa, volume 1, pages 43-50, 1999.

[18] W.E.K. Middleton. Vision through the atmosphere. University of Toronto Press, 1952.

[19] M. Sivak. The information that drivers use: is it indeed $90 \%$ visual? Perception, 26:1081-1089, 1996.

[20] C. Stauffer and W. Grimson. Learning patterns of activity using realtime tracking. IEEE Transactions on Pattern Analysis and Machine Intelligence, 22(8):747-757, August 2000.

[21] G. Welch and G. Bishop. An introduction to the Kalman filter. Technical Report TR 95-041, University of North Carolina at Chapell Hill, 2004. 